Citation: Mazur, I. (2020). A Functional Approach to Audio Description. Journal of Audiovisual Translation, 3(1), 226245.

Editor(s): A. Matamala \& J. Pedersen

Received: March 2, 2020

Accepted: October 12, 2020

Published: December 21, 2020

Copyright: (C2020 Mazur. This is an open access article distributed under the terms of the Creative Commons Attribution License. This allows for unrestricted use, distribution, and reproduction in any medium, provided the original author and source are credited.

\section{A Functional Approach to Audio Description}

\author{
Iwona Mazur ${ }^{\bowtie}$ \\ Adam Mickiewicz University
}

\begin{abstract}
The article discusses a functional approach to audio description (AD) and first proposes a classification of text types, followed by a model of source text (ST) analysis which encompasses three layers: the contextual, the macrotextual and the microtextual. The functional model helps identify the functional priorities in a given ST, which may then guide the audio describer's decision-making process: the results of contextual and macrotextual analyses will assist the describer in the selection of the so-called macro strategy, while the microtextual analysis may help in making lower-level decisions called micro strategies. Although the model has been designed primarily for didactic purposes, its principles may also be useful for more experienced describers. Additionally, the model constitutes a theoretical conceptualisation of $A D$ and attempts to better integrate $A D$ within the field of translation studies.
\end{abstract}

Key words: audio description, translation studies, functionalism, text types, skopos theory.

\footnotetext{
凶imazur@amu.edu.pl, https://orcid.org/0000-0002-7202-2239
} 


\section{Introduction}

In translation studies (TS) the 1980s saw a shift away from static linguistic and equivalence-based approaches to translation towards more flexible ones focused on the functions of the source text (ST) and the purpose (skopos) of the target text (TT) in the target culture (TC), which in turn determine the adopted translation strategies (e.g., Nord, 1997; Vermeer, 2000). As audio description (AD) is a type of translation, whereby images are translated into words, it could be assumed that most of the tenets of TS would be applicable to $A D$ as well. In the article I propose a functionally-oriented typology of audiovisual texts, which is followed by the proposal of a model that includes the contextual as well as macro- and micro-level analyses of the ST with a view to determining the functional priorities in AD. Such analyses will help inform the audio describer's decision-making process when selecting appropriate AD strategies (both at the macro and micro level) in order to best fulfil the skopos-or, as the case may be, various skopi- of $A D$ in a given context. The functional framework as applied to AD will first of all be an attempt to theorise about $A D$, but more importantly, it will be a practical tool for effective audio description, especially useful for trainee or beginner audio describers.

It should be noted, however, that due to the space limitations of a journal article only the basic premises of the AD functional model are outlined here. The model will be further developed and elaborated on elsewhere, including a more thorough linking to existing functional approaches to translation, other AD-based studies, and the study of multimodality. This in particular pertains to the model being a theoretical conceptualisation as well as a link between TS and AD. In this article the focus is on the proposal of a typology of audiovisual text types for AD purposes, as well as on ST analysis, both of which will also be further elaborated on in other publications. Nonetheless, every effort has been taken to ensure that the approach has been presented in as coherent a manner as possible.

\section{Functional Approaches in Translation Studies}

In the 1970s and 1980s, especially in Germany, there appeared approaches to translation that were centred around language functions and text types, the purpose of the $T T$, translation as an act of communication, and a more elaborate ST analysis, which all together have fallen under the umbrella label of functional approaches or functionalism. For example, Reiss (1989) proposes a classification of text types into informative (content-focused, e.g., a reference work), expressive (form-focused, e.g., a poem), operative (appellative-focused, e.g., an advertisement) and audio-medial (in which the other three functions are supported with images and sounds, examples being films or commercials, and which later acquired the label of "multimodal texts"). Reiss later removed the audio-medial text type from her classification, claiming that any audio-medial text would still belong to one or more of the other three categories, which in fact is an argument is favour of using her amended classification as a springboard for developing a more fine-tuned functional classification of multimodal texts as 
presented in section 4 below. Reiss (1989) suggests that "the transmission of the predominant function of the ST is the determining factor by which the TT is judged" (p. 109) and proposes translation methods governed by text type. Reiss and Vermeer (1984, as cited in Munday, 2001, p. 79), on the other hand, look at translation in terms of its skopos (i.e., "aim" or "purpose" from Greek) and state that translation methods are determined by the purpose for which a translation is produced.

Nord (1997) proposes a model for a translation-oriented ST analysis which involves "three aspects of functionalist approaches that are particularly useful in translator training" (p. 59): the importance of the translation commission or "brief" (e.g., the intended text functions, addressees, time and place of text reception, medium and motive), the role of a ST analysis (e.g., subject matter, cohesion, presuppositions, composition), and the functional hierarchy of translation problems ${ }^{1}$. Such an analysis helps to establish the functional priorities that guide the choice of the appropriate translation strategy. What is more, Nord (2006) makes a distinction between translation and translational action involving a number of players, much in line with the approach proposed by HolzMänttäri (1984, as cited in Munday, 2001, pp. 77-78).

The functional approaches as presented above build upon and complement each other and together constitute a comprehensive framework in which translation is set in a specific context, both as regards the ST to be translated (which has a specific author with their own intentions, cultural context and norms, etc.) as well as the TT, which is also placed in a specific socio-cultural context including the expectations of the client and the target audience (TA), their cultural background, as well as norms pertaining to the target language (TL) and culture. The functional approaches, however, are not without limitations. For instance, the strict distinctions between text types, or between form and content are often hard to maintain in practice ${ }^{2}$. Despite the criticism and being fully aware of it, the author of the present article stills sees the potential of the functional approach for AD purposes and proposes a model which is inspired by functionalism, but which also takes into account the unique and multimodal nature of $A D$. The issue will be further discussed in section 4 below.

\section{Functionally-oriented AD Research}

The idea of applying the functional approach to $A D$ has already been suggested by a number of $A D$ scholars. For example, Mazur (2014) draws analogies between the translation process and the AD process and suggests that some concepts underlying translation practices and theories could be usefully applied to $A D$, one of them being functionalism and the skopos theory. Along similar lines, Bardini (2017) claims that "Functional theory provides a solid framework for developing new audio description styles that deviate from conventional guidelines but take users' needs and source text's properties into account" (p. 67). Vercauteren $(2016$, p. 78 ) uses functionalism as a framework for his

\footnotetext{
${ }^{1}$ For a more thorough overview of the model see Munday (2001, pp. 82-84).

${ }^{2}$ For more critical claims also see Munday (2001, pp. 80-81).
} 
narrative approach to content selection in AD. He emphasises the importance of both ST analysis (a feature film in his case) and the needs of a visually impaired target audience. Starr (2017), in turn, employs a functionalist, skopos-oriented approach in a study concerning the usefulness of AD for children diagnosed on the autism spectrum who usually experience emotion-recognition difficulties and "mind-reading" challenges. Reviers (2017), on the other hand, conducts a corpus-based study of the language of Dutch AD, which she sets in the context of Systemic Functional Linguistics (SFL) (Halliday, 1994). She looks at AD primarily from the point of view of SFL categories such as the context of situation (field, tenor and mode), but also forms insights based on functional translation studies.

It should be added that functionalism underlies more studies within AD than the ones mentioned above (which refer to it explicitly). Many issues that are key to AD resonate with the functionalist approach, which is an important argument in favour of adopting this approach for AD. These issues include the importance of the TA and keeping their needs in the frame, moving away from formal equivalence-based criteria towards evaluating texts based on their adequacy for the TA, the key impact of context on AD strategies, or the importance of ST analysis (see e.g., Remael, Reviers, \& Vercauteren, 2014). All these approaches are the starting point for the development of the functional model of $A D$, the basic tenets of which are discussed below.

\section{A Functional Approach to AD: Proposal of a Model}

In this section a comprehensive $A D$ model is proposed which draws both on functionalist approaches to translation as well as previous AD research, as discussed above. Given the multimodal nature of both the ST and the TT (and the modal shifts that occur in the process of AD), the model is also inherently based on multimodality research, which presupposed that in a multimodal text meaning emerges through a combination of the visual, verbal and aural semiotic modes. A thorough analysis of a ST for AD purposes, as proposed here, would not be possible without understanding how the individual modes interact with each other and contribute to the overall meaning of the ST resulting in the so-called "multimodal cohesion", i.e., "integration and co-occurrence of different kinds of semiotic resources" (Van Leeuwen, 2005, p. 179). In AD a new multimodal text is created by translating intermodal links between images, sound and dialogue into intermodal links between sound and $A D$ and by creating the necessary intramodal verbal links between dialogue and $A D$. What also needs to be accounted for is the transfer of intramodal visual coherence into intramodal coherence in the AD by recreating the right visual cues in the description (Braun, 2011, pp. 650, 658).

The model is meant to be a theoretical conceptualisation of $A D$ (which will be elaborated on elsewhere), on the one hand, and a practical AD tool, on the other, and has been designed primarily for didactic purposes. The starting point for the proposed model is a classification of multimodal text types that has been inspired by Reiss' text typology (1989). The suggested classification is based on the identified predominant functions of multimodal texts that can be relevant from the point of view of $A D$ and includes five basic text types: informative, narrative, expressive, persuasive and 
entertaining (Fig. 1). The function of the informative text type is, as the name suggests, to inform. Examples of programmes under this category include: documentaries, news, cooking shows, travel programmes, interviews, educational TV, etc. The narrative text type includes genres that tell a story, such as films, TV series, soap operas, or comic strips. The expressive type is focused on the form and includes, for instance, art films or experimental cinema. The persuasive text type has an affinity to the operative text type in Reiss' classification and its purpose is to exert an influence on the viewer's behaviour through, for example, commercials or promotional materials. Finally, the entertaining text type includes all genres that fall under the umbrella of "light" entertainment, such as game shows, contests, reality shows, etc.

Figure 1.

The Classification of Multimodal Text Types for AD Purposes

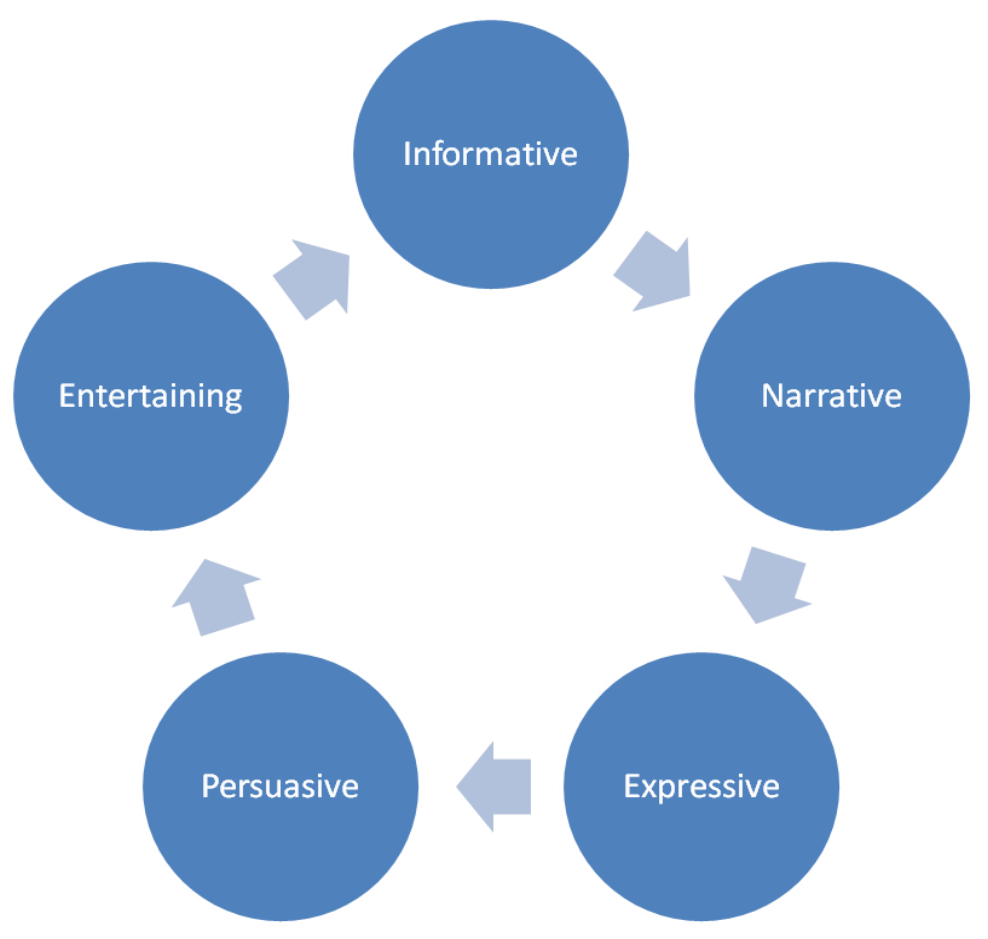

The division between the text types is not clear-cut, as they are fuzzy at the edges with often overlapping boundaries. In fact, hardly ever can we talk about "pure" text types (just as in the case of traditional written texts) and most of the time we deal with mixed types, with two or more functions present in one text, though more often than not the predominant function can be identified. For example, the line between the narrative and the expressive type is very thin, as on the one hand, regular feature films may have a very rich film language which would make them fall also under the expressive category, while on the other, an art film is most likely to tell a story. In a similar vein, a commercial may also tell a story or be visually appealing. A reality show may have some special effects or unusual camerawork that would make it fall not just under the entertaining but also the expressive category. And the entertaining function is likely to be exhibited to a greater or lesser degree by most of the multimodal texts in the other categories, as a travel programme or a cooking 
show in addition to being informative may also be entertaining. The same goes for a feature film or a TV series or even a commercial that contains humour.

It is also worth mentioning that text functions are dynamic and contextually-based and may change from ST to TT (e.g., a funny commercial that has a persuasive function for ST recipients may only have an entertaining function for TT recipients) or may be influenced by the channel of distribution (e.g., the same commercial may be aired on daytime television or at a film festival, for instance, in which case it will reach a narrower, but more 'targeted' group of potential customers). What is more, the way text functions are operationalised may depend on the background knowledge and personal aims of the TA. For example, a person may watch a wildlife documentary solely for the beautiful images rather than for the informative content. These issues will be elaborated on in the contextual and macrotextual analyses below. It should be noted that although here the functional approach to $A D$ concerns mostly film and television genres, its tenets are applicable to other AD types as well. For example, a theatre performance can be approached from the perspective of a more narrative or expressive text type, a sports event may be considered in terms of informative and entertaining functions, and a museum exhibition will surely combine informative and expressive functions.

\subsection{AD-oriented Functional Analysis}

The purpose of the functional analysis is to determine the functional priorities that would then guide the audio describer's choices as to which text functions and elements should be conveyed in the target text ${ }^{3}$. The proposed analysis is inspired by Nord's ST analysis for translation purposes (1991, 1997; also see section 2 above), though, due to the multimodal nature of the ST, the analysed aspects differ. Since Nord's model deals with texts in the traditional sense of the word (where the linguistic aspects are foregrounded), a number of modifications have been made to account for the unique type of transfer that AD involves (where images and sounds, rather than language, are the object of translation). Therefore, some of the analysed categories have been borrowed from Nord's model, new ones have been added and the very structure of the model has been reorganised to make the model more comprehensive for AD purposes.

The proposed functionally-oriented ST analysis model has three components, which are called contextual, macrotextual and microtextual. In the contextual analysis, as the name suggests, what gets examined is the context of the to-be-described text. This category includes the intended text function(s) of the ST which may be determined on the basis of the text type and genre, the addressees, time and place of text production and reception, the medium via which the ST is transferred and the one via which the TT will be transferred (e.g., television, an on-demand service,

\footnotetext{
${ }^{3}$ In the proposed model I use "target text" (TT) to denote an audio described product and "source text" (ST) to denote the product without $A D$, in line with translation-related terminology. Target audience (TA) means the intended users of $A D$, who include mainly-but not exclusively-persons with sight loss (PSL).
} 
a festival, etc.), and the purpose, i.e., why the text was produced and why it is being audio described. These elements roughly correspond to the elements of Nord's "importance of the translation commission" category described above. However, the contextual analysis also includes information about the ST that can be accessed without watching a film or programme, such as its synopsis, protagonists, creators, criticism or critical acclaim.

Having conducted the contextual analysis, the next step is the macrotextual analysis which happens while viewing a film or programme. Ideally, the viewing should first be "blind", i.e., without the image, which could be especially useful in a training set-up for students to learn to appreciate the original dialogues and sounds in a film in order to ascertain how much information an AD user can infer from the soundtrack itself and which essential sounds should not be covered with description or, as the case may be, would need to be described to be meaningful to the TA. This is followed by conventional viewing with both sound and image, and while doing so the describer should pay attention to all the details at the macro level which may be relevant for later $A D^{4}$.

As already mentioned, the ST analysis as presented below is firmly rooted in multimodality and requires a multimodal reflection every step of the way. It is thus imperative for those who would like to use the model to be familiar with how different semiotic modes contribute to the meaning-making processes in an audiovisual text (see e.g., Zabalbeascoa, 2008). Such a reflection would then help to determine how and to what extent the different modes support the overall function(s) of the text (as discussed above in section 4), making it possible to properly convey the functions in the AD.

To make the analysis more structured, I have divided the analysed aspects into content and form, though in reality it is usually the case that form and content mutually influence each other and may be difficult to separate for certain aspects.

Issues related to the content of the ST are:

- Title, credits, logo, text on screen.

- Subject matter: What is the film or show about? What does the title suggest about the subject matter?

- Contents: What are the contents of the ST? What is the cultural background for the ST? What is the general mood (e.g., for a film)? What is the leitmotif (for a reality show)? What is the main message (e.g., for a news broadcast)? What is being advertised and how (for a commercial)?

- Protagonists: Who are the main characters and what are the relationships between them?

- Temporal and spatial considerations: What are the temporal and spatial relations, how does a plot develop over time (for a film or a TV series). Is it linear? Are there any flashbacks or flashforwards? How many locations are there? What is the way that changes in location

\footnotetext{
${ }^{4}$ Also see the ST analysis-based approach to AD in Remael et al. (2014).
} 
and/or time are signalled in general terms (e.g., flashbacks could be depicted in black and white, while the present time in colour)?

- Dialogues, music and sounds: What information is conveyed through dialogues and other sounds that do not need to be described? Which portions of the dialogues would not be meaningful without the accompanying sounds? What role does music play in the ST?

Issues related to the form of the ST include:

- Structure: How is the content organised (e.g., in a reality show, does the content follow a specific pattern)? How is the presented information structured? In a film, what are the tools for local (within a scene) and global (between scenes) coherence (Braun, 2007, pp. 365-366)?

- Language: What language type or variety is used in the ST? Does the language match its genre? Do the protagonists speak a vernacular or use slang expressions or taboo language? Is there any specialised vocabulary (e.g., in a documentary or a science show)? This will give us an indication as to what language style to use in the $A D$ to make it consistent with the ST language.

- Filmic language: What camerawork has been used in the ST and how functionally relevant is it for its reception and/or appreciation? This may be an indication as to whether to include instances of filmic language in $A D$ or reflect the narrative function of given filmic techniques in the description (see e.g., Kruger, 2010; Fryer \& Freeman, 2012).

- Sound: How does sound influence the structuring of a film, e.g., in terms of editing, temporal development, cohesion, etc.?

- Time constraints: How dense are the dialogues? Are there any moments in the ST that will allow for more $A D$ ? This will give us a general indication of how much time we have for $A D$, whether we would need to use the foreshadowing technique (i.e., describe ahead of time) in the cases where scenes start immediately with a dialogue or if we may add some information later, when there is more time for AD.

Issues related to both form and content:

- Text functions: Based on the macrotextual analysis of both the form and content of a multimodal text, the functions of the ST are determined. While the contextual analysis helps determine the primary function based on the text type, the macro analysis may help pinpoint any additional secondary functions the ST may exhibit. What also gets decided here, taking into account the results of the contextual analysis, is whether the ST function(s) will remain the same for the TA or whether they will change.

- Presuppositions: Based on the TA's presumed knowledge, can we assume that the presented information will be clear to them? For example, if the ST includes a cultural reference, can we assume that it will be clear to the majority of the TA or will the reference need further elaboration or-on the contrary-generalisation? 
While the idea behind the macrotextual analysis is to get the general idea of a programme's form and content, the micro functional analysis involves looking at a specific $A D$ unit, be it a scene or even a take, while describing and determining what the functions of the visual (and auditory) elements are and how relevant they are for the TA to understand or appreciate the programme. These should be related to the functions identified in the contextual and macrotextual analysis. Hence, the microtextual analysis will use some of the same parameters used in the macro analysis, such as time and space considerations (e.g., the way that change in time or location is signalled in individual scenes), characters (e.g., what is the most relevant information to mention about them in a given scene, is it their looks or actions or reactions), dialogues and sounds (what specific information is missing for a dialogue to be meaningful without visuals, which sounds would need to be described for multimodal cohesion and which will be clear without description), filmic language (does a particular instance of filmic language merit inclusion in the AD script and how), etc.

Usually, in a complex visual scene a number of elements compete for the attention of the viewer. And while the sighted viewer can absorb a given scene holistically in a non-linear manner, the AD user will have to process the description in a linear way. For this reason, the describer needs to make a selection of the most salient elements. Kruger (2012) distinguishes between visually-salient and narratively-salient elements, while the proposed functional model would rather operate on the basis of functional saliency, i.e., the importance of a given element taking into account the function it has in a given scene or take. For instance, it could be that the same culture-specific item would be functionally salient in a travel programme, but would only serve as an insignificant prop in a reality show.

Figure 2 presents the three layers of the functional analysis. 
Figure 2.

The Three Layers of the Functional Analysis

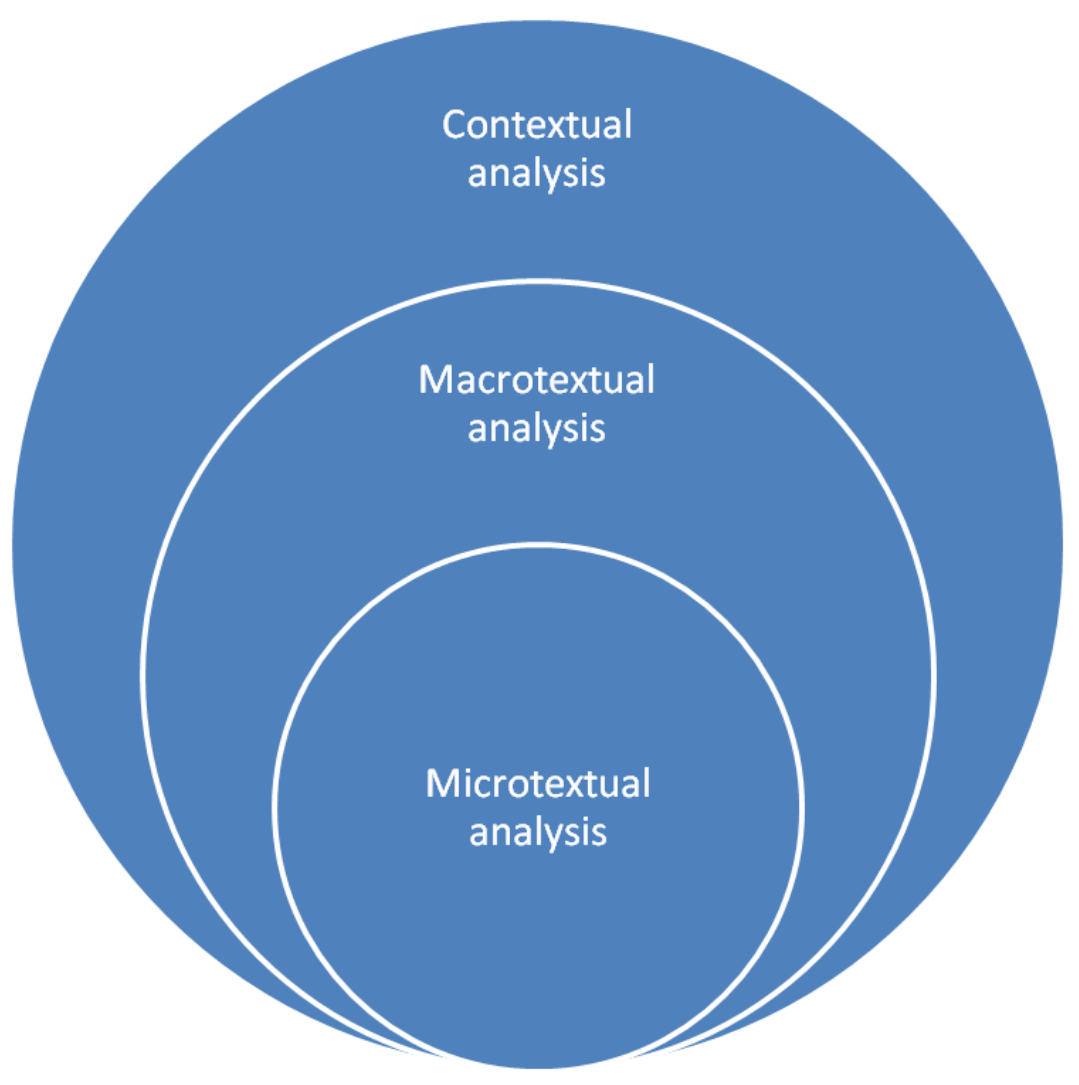

\subsection{From the Functional Analysis to Functional Priorities and AD Strategies}

The three-tier analysis will thus help determine the functional priorities which will then guide the describer's choices regarding both the content and the style of AD. Below I propose a hierarchy of AD-related questions, akin to Nord's (1997) hierarchy of translation problems, the answers to which will be influenced by the identified functional priorities.

1) What is the primary function of the AD? Are there any secondary functions? Is this related to the intended purpose (skopos) of the AD? Is the AD supposed to inform, entertain, or influence the choices of the TA?

2) Based on the intended purpose, what is the adopted macro strategy towards the AD?

3) Which elements will have to be explained to the TA based on their presumed knowledge and the intended purpose of the AD?

4) What will the micro strategies be?

While the contextual and especially the macrotextual analyses help determine the global or macro strategy for the whole text (cf., Jääskeläinen, 1993; "initial norm" Toury, 1995; "domestication vs. 
foreignisation" Venuti, 1995, for translation), the microtextual analysis would give an indication as to the local or micro strategies (cf., Jääskeläinen, 1993; "translation procedures" Newmark, 1988 for translation), i.e., lower-level decisions that are applied in the case of individual audio description crisis points (Mazur, 2017).

For example, if based on the contextual and macrotextual analyses it is determined that a text is an informative one, the macro strategy may be to focus on conveying the contents as much as possible. In the case of a TV series that tells a story or stories, the functional priority will be narration so the $A D$ macro strategy will be to re-create the story by describing the essential visual elements so that the $A D$ along with the original soundtrack constitute a coherent whole (see e.g., Vercauteren, 2016). If, on the other hand, a given production falls under the expressive category (e.g., a film with a very rich and unusual filmic language), the describer may decide to focus on rendering the artistic dimension in $A D$ as well (though, in the case of a conflict, the narrative function prevails, as the viewers need to be able to follow the story in the first place). For a persuasive text, such as a commercial, the macro strategy will be to convey the persuasive function in order to encourage the $A D$ user to buy the advertised product or service. Finally, the macro strategy for an entertainment show will be to emphasise in $A D$ those elements that make a given show entertaining, for example by using humorous language in the $A D$, if the show is supposed to make the audience laugh. The adopted macro strategy will guide the describer's lower-level decisions as to what and how to describe, though it should be noted that although the macro strategy guides the choice of the subsequent micro strategies, it does not determine them, as they always need to be negotiated individually. Also, some of the information identified both in the contextual and macrotextual analyses may be included in an audio introduction ( $\mathrm{Al}$ ), should a given audiovisual production have one $^{5}$.

In the section below a practical application of the functional model will be presented.

\section{AD-oriented Functional Analysis: A Case Study}

In this section the functional analysis as described above will be applied to break down the first episode of a cooking show Nigella Bites (2001) for AD purposes. The contextual, macrotextual and microtextual analyses of the programme will be discussed and so the focus will be on the text functions that would need to be conveyed in the AD.

\subsection{Contextual Analysis}

Nigella Bites is a cooking show so we may assume that its main function is an informative one. Therefore its purpose is to provide the viewers with information on how to cook and the target

\footnotetext{
${ }^{5}$ For what information to include in an Al see, for example, Fryer \& Romero-Fresco (2014).
} 
audience will then mainly be persons interested in cooking, most likely with some knowledge of cooking fundamentals. We can assume the audio described version of the show will have the same purpose and type of addressees. The show was broadcast on Channel 4 in the UK between 20002001, so if we were to describe it today some of the information may be dated and may require explanation. Also, it is important to consider the culture to which the intended AD target audience belong. Do they live in the UK or not? If not, some concepts like UK-specific ingredients may need explaining. As for the medium, the original show was aired on television, whereas if we were to describe it now, it would most likely be available on DVD or an on-demand platform.

Research on the show's host would yield the following information. In addition to being a cooking show host, she is also a cookbook writer (that is why in the show sometimes we see her during a photo shoot or writing on a computer). The programme was filmed in Nigella's London home. At the time of filming the programme Nigella was married to John Diamond who suffered from throat cancer (diagnosed in 1997, he died in 2001). They had two children, a daughter Cosima and a son Bruno. This information may help the describer interpret the ST as both the husband (who hardly says anything) and the children appear in the show.

Also, a quick look at the reviews ${ }^{6}$ of the show can give the describer an indication as to what to expect from it.

Nigella Bites is an indisputably sensual addition to the canon of cookery programmes. After fearsome Fanny Craddock, dowdy Delia Smith, jolly Jamie Oliver, here is naughty Nigella Lawson. For her, the whole point of cooking is the "pleasure" of food. She espouses uncomplicated recipes for a modern generation constantly on the go and gives short shrift to dietary considerations. (...)

As the parodists were quick to point out, there is something of a sexual sub-text to Nigella Bites, as much in the lingering attention on the presenter herself as [in] the unashamed sensuality of its recipes. Watch in rapture as the camera scans up and down Nigella's curvaceous but not too well-nourished figure, zooms in on her luscious lips, follows her into her store cupboard to "see my chocolate stash", or wobbles in sympathetic ecstasy as she applies lashings of cream or expertly spatchcocks a chicken. In short, whether you're an envious female wondering how Nigella manages to juggle a hectic life and still produce such delicious culinary fare, or a couch potato male who can barely boil an egg, chances are you'll find much to delight in here. (...)

The two reviews above suggest that Nigella Bites is more than just a regular cooking show. What is emphasised in them is the aesthetic dimension: the sensuality of cooking ("an indisputably sensual addition to the canon of cookery programmes") and the pleasure of eating ("the whole point of cooking is the 'pleasure' of food"), as well as the attractive, at times even seductive, image of the host ("there is something of a sexual sub-text to Nigella Bites", "naughty Nigella Lawson"). There is

\footnotetext{
${ }^{6}$ https://www.amazon.co.uk/Nigella-Lawson-Bites-DVD/dp/B00006G9X2
} 
also a hint as to how the sensual effect has been accomplished with the special use of camerawork, such as close-ups, ("[the camera] zooms in on her luscious lips") or lingering shots ("the camera scans up and down Nigella's curvaceous ... figure", "[the camera] follows her into her store cupboard"). What is more, there is an indication as to the target audience of the show: both men and women are likely to enjoy the show.

Although it may seem that the contextual analysis is laborious and perhaps superfluous, all of the above information could be gathered from practically viewing the Wikipedia and Amazon webpages and in fact should not take the describer longer than several minutes. The information sets the stage for the second analysis and will surely help the describer better grasp all of the ins and outs of the programme and pay attention to all the relevant details. What is more, it may even give the describer an indication as to what kind of language to use to describe the host and her actions.

\subsection{Macrotextual Analysis}

Having conducted the contextual analysis the next step is to carry out the programme's macrotextual analysis by viewing it in its entirety and paying attention to all the AD-relevant elements both in terms of form and content.

Below is the analysis of the relevant details of the first episode of Nigella Bites:

Issues related to the content of the ST:

- Title, credits, text on screen: The show starts with the title (Nigella Bites) displayed over simple animation with music, followed a bit later by the title of the episode Fast Food. This suggests the main theme of the episode: food prepared on the go. The end credits roll very fast over a dinner scene, so there will be little time to read them out. The show does not end with the end credits, though: after they finish there is the final scene of Nigella's night-time visit to the refrigerator, where she eats some of the leftover food. The show ends with the logo of the production company: PACIFIC: A Pacific Production in association with Ricochet for Channel Four.

- Subject matter: The show presents a number of recipes to be made on the go. This is also suggested by the title of the episode: Fast food.

- Contents: The core of the episode are six recipes that Nigella presents in her kitchen: linguine, tomato soup with rice, salmon with mushy peas, affogato, chocolate pudding and marinated chicken. These should get priority in the description in order to fulfil the informative function of a cooking show. Here we should look out for any information that may be missing in order for the AD user to be able to recreate the recipes. As it turns out, most of the recipes are "narrated" by the host, from the ingredients to the preparation process to the actual serving of a meal. The recipes are interspersed with snippets of Nigella's busy life: she takes her son to the nursery, does a photo shoot, picks her son up from the nursery and her daughter from school, goes shopping with them, serves them a snack for teatime, gives them a bath. 
- Protagonists: The main protagonist who should get most attention in terms of description is obviously the show's host. However, in the episode we also see her children: her son Bruno and her daughter who is not named in this particular episode. Interestingly, at the after-school teatime scene, there are three children at the table. Again, thanks to the contextual analysis we will be able to assume that only two of them are Nigella's. Other persons in this episode include some photographers, a prop stylist, a make-up artist, etc., during a photo shoot scene, as well as Nigella's three friends (two female, one male) at the dinner scene at the end of the show, all of whom need not get extensive attention in the AD.

- Temporal and spatial considerations: The main shooting location is Nigella's kitchen in her home in London (as we know from the contextual analysis). It is mostly daytime/late afternoon, except for the night-time visit at the fridge at the end of the episode. The indoor scenes are interspersed with outdoor ones, shot on the streets of London, in a shop, and at a café where the kids choose their desserts after school. The time orchestration is linear.

- Dialogues, music and sounds: There are very few dialogues, and if they are present they are rather insignificant (e.g., some remarks exchanged with the photo shoot staff), which means that, if need be, they could potentially be covered with AD. The most important elements are Nigella's monologues when presenting the recipes, which should be left intact in the AD. There are also sounds of food being prepared (e.g., salmon sizzling on a grill pan or chocolate being crumbled), which - if possible-should not be talked over entirely, as they too (like the camerawork discussed below) emphasise the sensual undertones of cooking. Some scenes are accompanied by instrumental music, which is a good place to insert $A D$, though it should not be talked over entirely, as the music too enhances the visual scenes. There were only four instances identified in the narration where AD would be indispensible for cohesion purposes, as without it the audience would not be able to make sense of what is being said. One such instance is when Nigella is licking ice cream on a cone in her garden during a photo shoot and says "I feel like I am in one of those bad awful English porn films of the seventies". Without $A D$, the audience would be puzzled, as this is an intriguing statement and there is no immediate context that would help make out its meaning.

Issues related to the form of the ST:

- Structure: The show follows a consistent pattern: the presentation of the recipes is intertwined with scenes depicting the host's real life.

- Language: Lawson uses very vivid language when describing food, with lots of adjectives (velvety, rich, smooth), which corresponds to the sensual nature of her cooking. A similar language style could thus be adopted in the AD.

- Filmic language: The contextual analysis above has already indicated the special use of the camerawork in the show, especially in the form of close-ups. This is confirmed by the macrotextual analysis: the episode in question abounds in close-ups of both the food that is being prepared (coffee being poured over ice cream in a bowl to make an affogato or a hand 
pressing a knife blade and crushing a clove of garlic) as well as of the host herself (her face, eyes, lips, hands). At other times the camera follows Nigella around, as if sneaking up on her, for example when she walks down to the refrigerator at night. Some images are out of focus to become sharp only after a while, making a given scene appear somewhat dreamy. This style of filming gives rise to the sensual connotations of cooking that have already been identified in the contextual analysis, which are further reinforced by the presentation of the host herself (her figure hugging blouses emphasising her large bosom, her finger licking, the way she murmurs when she tastes food, etc.). In fact, she does compare herself to a porn star when licking ice cream (see above), which suggests that this is a consciously built image. All this points to the fact that the expressive function is realised in the show, which should also be reflected in the AD.

- Sound: There is theme music which marks the beginning and end of the show and a portion of which is used to separate "chapters" or "sections" of the episode with different recipes.

- Time constraints: The show is uneven in terms of time constraints: in the parts where Nigella narrates the recipes it is very dense, leaving little room for description. In the other parts, there is music, other sounds and sporadic dialogues, which affords more time for AD.

Issues related to both form and content:

- Text functions: The informative function of the show as realised through the presentation of the recipes is predominant; however, watching the entire episode will make it clear that it is not the only one. As already mentioned, Nigella's cooking is interspersed with scenes of her rather hectic life. In fact, we seem to follow her over a couple of her busy days, as if we were watching a story, so it is clear that the show also fulfils a narrative function which will also have to be reflected in the AD for the TA to have the complete experience of the show. This is in fact a backdrop to the recipes which are meant to be quick and easy, ones that even a very busy working mum can prepare in between her many chores, which is also emphasised by the episode's title Fast food and the host herself in the short introduction at the start of the episode. Finally, as identified in the analysis of the camerawork above, the expressive function plays an important part in how the show is received, especially as regards the sensuality of cooking and the host herself. In terms of the multimodal distribution, the informative function relies mostly on the host's monologues and as such is more readily accessible to $A D$ users, whereas the visuals mainly contribute to the expressive and narrative functions. So even if these are not the "main" functions of the text, they might be the main aspects to focus on in the AD.

- Presuppositions: We can suppose that the persons watching the show are interested in cooking and will know basic cooking techniques.

One of the advantages of the macrotextual analysis is that we can determine what will be clear to the TA from the context, as sometimes things can get explained later on, and an earlier description may turn out unnecessary. 


\subsection{Microtextual Analysis}

As already mentioned, the microtextual analysis happens while describing a given take or scene, and with practice its principles should become internalised and occur almost automatically. The main idea here is, again, to assess the constituents of a given scene in terms of the functions they fulfil and thus determine the functional priorities that need to be described in order for the scene to be intersemiotically cohesive and meaningful for the TA (see examples of some micro strategies below). Just to give an example, the show starts with an introduction to the episode by Nigella sitting inside, in an armchair, talking about the importance of meals that are quick and easy to prepare. This is followed by a scene in which the host brings her son to the nursery. In terms of time and space considerations, it is daytime (the context will indicate to us that it is morning) and the scene is shot first in a car, and then outside on the street. There are just the two characters. There is instrumental music in the background with a fairly fast tempo. In terms of filmic language, the individual shots are short and the cuts fast (conveying the impression of being in a hurry), and there are occasional closeups (emphasising Nigella's slightly stressed face). There is practically no dialogue, just one line said by Nigella ("I am bringing Bruno to the nursery a bit late."), which reinforces the message conveyed by the other two semiotic modes (the quickly changing visuals and the fast music) and brings the meaning of the scene together: they are running late, Nigella is a busy mother, etc. This short analysis helps determine the functional priorities for the scene (focus on the actions, the narrative function), which are elaborated on in the next section along with the discussion of possible AD solutions.

\subsection{Functional Priorities and AD Strategies}

Based on the functional analysis above, we can determine both the primary and secondary functions of the first episode of Nigella Bites. Since we are dealing with a cooking show, where the main aim is to provide the audience with information on how to prepare meals according to the presented recipes, we can assume that the primary function will be informative. However, as the show also involves an aesthetic dimension expressed in the form of the host's sensuality and her sensual approach to cooking, as emphasised by the use of special filmic techniques, the secondary function is an expressive one. Finally, the programme presents stories from Nigella's personal life, which implies the narrative secondary function.

Identified functional priorities will then inform the selection of the macro strategy, which more often than not corresponds to the primary function (or skopos) of the to-be-audio-described programme. In this case, given that we are to describe a cooking show, the skopos of the original show is to inform the viewers about a selection of recipes to be recreated in the comfort of their own kitchens. It is likely that the skopos will remain the same for the audio described show-AD users should also be able to replicate the presented recipes. It seems therefore reasonable to posit that the macro strategy for audio describing the show will be content preservation: in the case of elements 
competing for description, the ones that help convey the contents or factual information making the presented recipes usable, should be prioritised.

However, since the functional analysis revealed two other (secondary) functions, i.e. the expressive and the narrative, the micro strategies should help reflect them too, if possible. For example, the snippets of Nigella's busy life at the start of the episode can be described in a way that conveys the narrative function of this part, so the focus will be on the actions, bearing in mind that the purpose of this part is to emphasise the hostess's hectic life which sets the scene for the "fast food" recipes that follow. So here the describer may use short, simple (possibly verbless) clauses that correspond to the quickly changing scenes, e.g., "In a car. Nigella driving, a small boy on the back seat. She takes him out of the car. They walk quickly. Nigella speaks through the intercom ('I am bringing Bruno to the nursery a bit late')." On the other hand, later on in the episode there is a photo shoot scene featuring a layered chocolate cake, which exhibits more of an expressive function. Here the describer may decide to focus more on Nigella's look or the camerawork (e.g., by reflecting the close-ups in the language of the $A D$ through placing what is in the close-up in the sentence-initial position and using the passive voice if necessary, e.g., "Thick cream gets slathered on the first layer of the cake"). Here, the AD may be more "leisurely" and full of adjectives to translate the aesthetic dimension of this highly visual scene into words.

\section{Limitations of the Functional Approach to AD and Further Research}

The presented functional model for analysing a ST for AD purposes may seem complex and its application time-consuming. However, it should be remembered that the model is first and foremost meant for training purposes to help learners "break down" a complex multimodal text and understand the functions the relevant constituents have in the text so that they can be properly reflected in the $A D$. Also, the very awareness of the proposed text types can help learners (but also professional describers) in prioritising the information that needs to be conveyed in the AD. Another limitation of the model may be that often we deal with mixed text types, rather than pure ones. This is also one of the major criticisms that the original functional approach to translation received. And while it cannot be denied that often we do have a mixture of a number of text functions, usually the prevailing one can be determined, plus the other functions are also useful in guiding the choice of micro strategies. Finally, it may seem that the model may be useful mainly in the case of ADs produced at the post-production stage, rather than within the paradigm of accessible filmmaking (Romero-Fresco, 2019), although this is not entirely true. While some components of the contextual analysis (e.g., the critical acclaim of a film) may indeed only apply in the former case, the other tenets of the approach may be useful also in accessible filmmaking, as the describer could still look for the functional priorities and emphasise them in the AD. And finally, the model needs not be applied in its entirety-any of its constituents is likely to work on its own, for example the contextual analysis may be done independently without the need to do the other two types of analyses. 
As already mentioned, these are just the basic tenets of the model, as it could not be presented here in its entirety due to spatial constraints, so at times it may seem not thoroughly explained. The missing links will be discussed in separate publications, especially as regards the theoretical conceptualisation, a more thorough linking with multimodality and functional approaches to translation as well as an elaboration of the three tiers of the analysis along with its individual components. The model offers potential for further research, for instance by being modified and adapted for the purposes of other AD types, such as theatre or museum AD. It could also be validated by being applied in actual AD training.

\section{Conclusion}

The primary purpose of developing the functionally-oriented $A D$ model as presented above has been to provide a theoretical framework for the study of $A D$ and to integrate $A D$ within the scope of translation studies as a discipline, by positing that $A D$ is a type of translation to which the tenets of the functional approach can be applied as well. Although the model is grounded in translation theory, it is very much applicable to the practice of $A D$. It can be especially useful to train prospective audio describers, as it can take a lot of pressure off trainees in particular, who can often feel overwhelmed by the amount of visual information that they think needs to be fit into the short available time. As here the focus is on making the AD fulfil its intended function(s), such as to convey information, tell a story or make the audience laugh, it means that we do not need to describe "everything." It should, however, be emphasised that the approach is by no means a "minimal" one, where we just focus on conveying the main functions. Rather, it is an orientation tool for prioritising information that may compete for our attention, according to the intended functions or purposes of a given text. However, should there be enough time, more details may by all means be included in the AD that would give the audience an even fuller experience of the described programme. The advantage of the presented model is that it is compatible and can be easily integrated with other AD approaches such as the narratological approach to content selection (Vercauteren, 2016). Although the model is finegrained, it needs not be applied in its entirety-any of the layers of the functional analysis can in fact be applied independently, or even certain selected elements can be used to facilitate the process of analysing a text for AD purposes. In fact, just the very awareness of the different text types and their related functions can be enough of a guiding tool for describers in their decision-making process and the selection of appropriate $A D$ strategies. As such, it can also be useful for more seasoned describers, especially ones with backgrounds other than in translation. 


\section{References}

Bardini, F. (2017). Audio description style and the film experience of blind spectators: Design of a reception study. Rivista Internazionale di Tecnica della Traduzione, 19, 49-73.

Braun, S. (2007). Audio description from a discourse perspective: A socially relevant framework for research and training, Linguistica Antverpiensia NS, 6, 357-369.

Braun, S. (2011). Creating coherence in audio description. Meta: Translators' Journal, 56(3), 645662.

Dulay, S. (Producer), \& Warwick, B. (Director). (2001). Nigella Bites [TV series]. Country of origin: United Kingdom. Production companies: Flashback television/Pacific.

Fryer, L., \& Freeman, J. (2012). Cinematic language and the description of film: Keeping AD users in the frame. Perspectives: Studies in Translatology, 21(3), 412-426.

Fryer, L., \& Romero Fresco, P. (2014). Audiointroductions. In A. Maszerowska, A. Matamala, \& P. Orero (Eds.), Audio description: New perspectives illustrated (pp. 11-28). Amsterdam: John Benjamins.

Halliday, M. A. K. (1994). An introduction to functional grammar. London: E. Arnold.

Holz-Mäntttäri, J. (1984). Translatorisches Handeln: Theorie und Methode [Translatorial action: Theory and methodology]. Helsinki: Suomalainen Tiedeakatemia.

Jääskeläinen, R. (1993). Investigating translation strategies. In S. Tirkkonen-Condit \& J. Laffling (Eds.) Recent trends in empirical translation research (pp. 99-120). Joensuu: University of Joensuu Press.

Kruger, J.-L. (2010). Audio narration: re-narrativising film. Perspectives: Studies in Translatology, 18(3), 231-249.

Kruger, J.-L. (2012). Making meaning in AVT: Eye tracking and viewer construction of narrative, Perspectives: Studies in Translatology, 20(1), 67-86.

Mazur, I. (2014) Projekt ADLAB i funkcjonalizm w przekładzie - w stronę strategii audiodeskrypcyjnych [The ADLAB project and functionalism in translation - towards audio description strategies]. Przekładaniec. A Journal of Translation studies, 28, 11-22.

Mazur, I. (2017). Audio description crisis points: The idea of common European audio description guidelines revisited. In J. Díaz-Cintas \& K. Nikolić (Eds.), Fast forwarding with audiovisual trans/ation (pp. 127-140). Bristol: Multilingual Matters.

Munday, J. (2001). Introducing translation studies. London: Routledge.

Newmark, P. (1988). A textbook of translation. New York: Prentice Hall.

Nord, C. (1991). Text analysis in translation: Theory, methodology and didactic application of a model for translation-oriented text analysis. Amsterdam: Rodopi.

Nord, C. (1997). Translating as a purposeful activity. Functionalist approaches explained. Manchester: St. Jerome Publishing.

Nord, C. (2006). Loyalty and fidelity in specialised translation. Confluencias-Revistas de Draducao Cientifica e Tecnica, 4, 31-41.

Reiss, K. (1989). Text types, translation types and translation assessment (translated by A. Chesterman). In A. Chesterman (Ed.), Readings in translation theory (pp. 105-115). Helsinki: Finn Lectura. (Original work published in 1977).

Reiss, K. \& Vermeer, H. (1984). Grundlegung einer allgemeinen Translationstheorie [The fundamental principles of a general translation theory]. Tübingen: Niemeyer.

Remael, A., Reviers, N., \& Vercauteren, G. (Eds). (2014). Pictures painted in words. ADLAB audio description guidelines. Trieste: EUT. 
Reviers, N. (2017). Audio description in Dutch: A corpus-based study into the linguistic features of a new, multimodal text type (Unpublished doctoral dissertation, Universiteit Antwerpen, Antwerp). Romero-Fresco, P. (2019). Accessible filmmaking: Integrating translation and accessibility into the filmmaking process. London: Routledge.

Starr, K. L. (2017). Thinking inside the box: Bespoke audio description for cognitively diverse audiences. Paper presented at the 6th Advanced Research Seminar on Audio Description ARSAD, March 16-17, Barcelona, Spain.

Toury, G. (1995). Descriptive translation studies and beyond. Amsterdam: John Benjamins. Van Leeuwen, T. (2005). Introducing social semiotics. London: Routledge.

Venuti, L. (1995). The translator's invisibility: A history of translation. London: Routledge. Vercauteren, G. (2016). A narratological approach to content selection in audio description (Unpublished doctoral dissertation, Universiteit Antwerpen, Antwerp).

Vermeer, H. J. (2000). Skopos and commission in translational action. In L. Venuti (Ed.), The translation studies reader (pp. 221-232). London: Routledge. (Original work published in 1989).

Zabalbeascoa, P. (2008). The nature of the audiovisual text and its parameters. In J. Díaz-Cintas (Ed.), The didactics of audiovisual translation (pp. 21-37). Amsterdam: John Benjamins. 